\title{
Prediction of Caregiver Burden Based on Sense of Coherence and Psychological Capital in the Spouses of Amputee Veterans
}

\section{ART ICLE INF O}

\section{Article Type}

Descriptive Study

\section{Authors}

Dargahi Sh. ${ }^{1} M S C$,

Sheykholeslami A. ${ }^{1} P h D$,

Azimi D.* $M S C$,

Shooani E. ${ }^{2} M S C$

How to cite this article
Dargahi Sh, Sheykholeslami A, A-
zimi D, Shooani E. Prediction of
Caregiver Burden Based on Sen-
se of Coherence and Psychological
Capital in the Spouses of Amput-
ee Veterans. Iranian Journal of War
\& Public Health. 2018;10(4):203
-208.

*Educational Sciences Department, Educational Sciences \& Psychology Faculty, University of Mohaghegh Ardabili, Ardebil, Iran

${ }^{1}$ Educational Sciences Department, Educational Sciences \& Psychology Faculty, University of Mohaghegh Ardabili, Ardebil, Iran

${ }^{2}$ Consulting Department, Educational Sciences \& Psychology Faculty, University of kharazmi University, Tehran, Iran

\section{*Correspondence}

Address: University of Mohaghegh Ardabili, End of University Street, Ardebil, Iran. Postal Code: 5619911367

Phone: +98 (45) 31505000

Fax: +98 (45) 33512200

dazimi038@gmail.com

\section{Article History}

Received: April 10, 2018

Accepted: August 04, 2018

ePublished: October 11, 2018

\section{A B S T R A C T}

Aims One of the damaging factors of the family structure is the disability of one of its members, which can be due to events such as war. Due to the need to pay attention to the members of the veterans' family, especially their spouses, the aim of this study was to predict the caregiver burden based on sense of coherence and psychological capital in the spouses of amputee veterans. Instruments \& Methods This descriptive-correlational study was conducted in 2016 among spouses of amputee veterans in Piranshahr. Using available sampling method, 120 people were selected as sample. For data collection, Pai and Kapur's Family Burden Interview Scale, Antonovsky's Sense of Coherence Scale, and Luthans's Psychological Capital Questionnaire were used. The data were analyzed, using SPSS 18 software, Pearson correlation, and stepwise regression test.

Findings There was a negative and significant correlation between caregiver burden and sense of coherence $(r=-0.28 ; p<0.01)$ and psychological capital $(r=0.41 ; p<0.01)$. The sense of coherence was significantly able to predict about $7 \%$ of the variance of caregiver burden in veterans' spouses $(\mathrm{p}<0.005)$. Also, self-efficacy in the first step and resilience in the second step significantly predicted $14 \%$ and $17 \%$ of the variance of the caregiver burden of the spouses of veterans, respectively $(\mathrm{p}<0.001)$.

Conclusion The sense of coherence and psychological capital reduce caregiver burden in the spouses of amputee veterans.

Keywords Sense of Coherence; Spouses; Amputation; Veterans

\section{I T A T I O N L I N K S}

[1] Psychiatric symptoms in chemical and somatic ... [2] The effectiveness of training stress coping strategies on marital ... [3] Assessment of relationship quality of life and coping skills in spouses of chemical devotees ... [4] Level of expressed emotion, depression and caregiver burden in wives ... [5] The relationship among depression and caregiver ... [6] Psychological and marital distress in spouses of ... [7] Military-related post-traumatic stress disorder ... [8] Stress Management and Resilience Training (SMART) program to decrease stress and enhance ... [9] Sense of coherence and attrition during four-year follow-up in cohorts ... [10] Effectiveness of training the review of life on life satisfaction and sense of coherence ... [11] The structure and properties of the sense of coherence ... [12] Study of causal relationship between sense of coherence, psychological ... [13] Factors predicting sexual functioning in patients 3 months after surgical procedures for breast cancer ... [14] Role of psychological capital in academic burnout of ... [15] Fishing for happiness: The effects of generating ... [16] The relationship between the hopefulness, daily ... [17] The causal relationship between psychological capital and positive emotions ... [18] Association between family caregivers' quality of life and psychological ... [19] Analytical on effect of resilience interventions on mental health in family caregivers ... [20] Self- efficacy and caregiver strain in alzheimer's ... [21] The relation between psychological capital and ... [22] Role of psychological capital in academic burnout of ... [23] Comparative evaluation of psychological burden in ... [24] Validation of Sense of Coherence (SOC) 13-item scale in Iranian ... [25] The relationship between psychological capital, hope for employment and academic ... [26] Community resilience and sense of coherence as protective... [27] Role of self-compassion and sense of coherence... [28] Enhancing sense of coherence via early intervention among ... [29] The mediating role of psychological capital on the association between ... [30] Quality of life of the spouses of war related amputees ... [31] The development and resulting performance impact ... [32] The Role of Psychological ... [33] Investigating causal relationships between gender ... [34] The relationship of job burnout, perceived social ... 
فشار روانى مراقبت در همسران جانبازان بالا ززارش شده است[4].

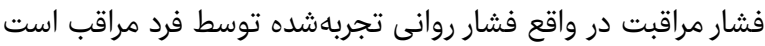

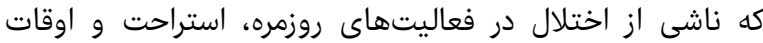

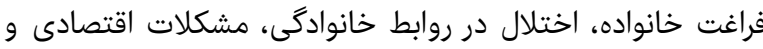

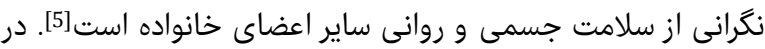

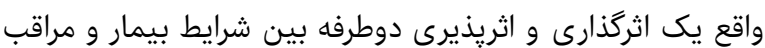

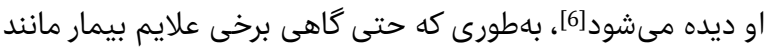

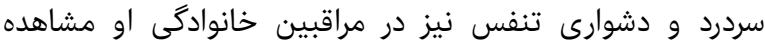

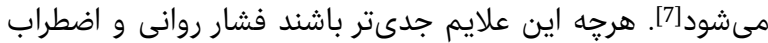

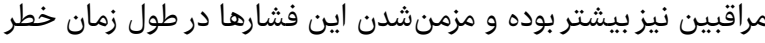

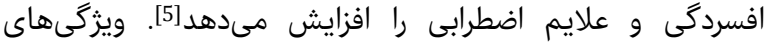

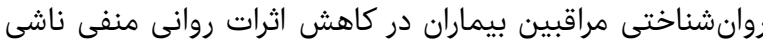

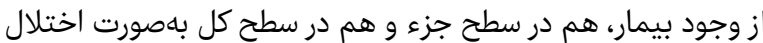
در كاركردهاى سيستم خانواده موثر است.

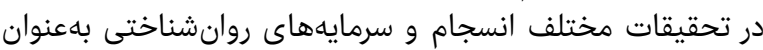

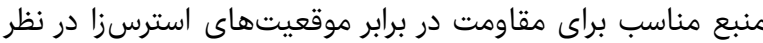

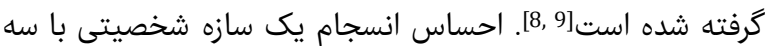

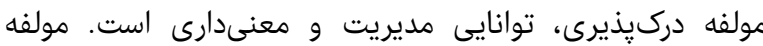

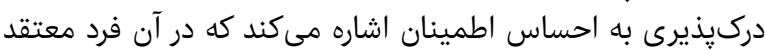

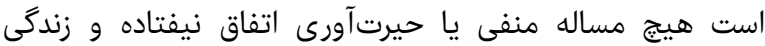

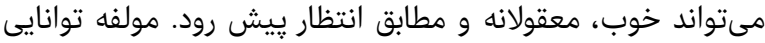

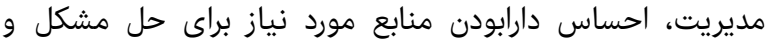

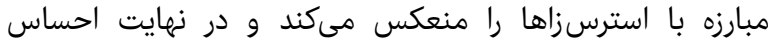

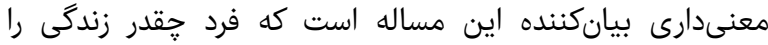

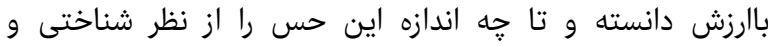

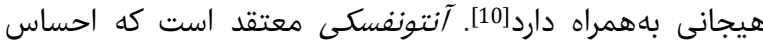

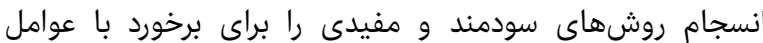

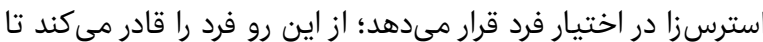

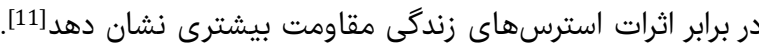

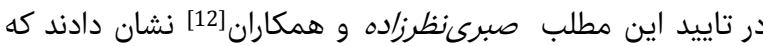

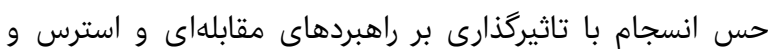

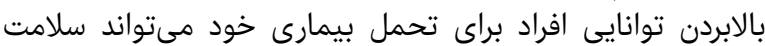

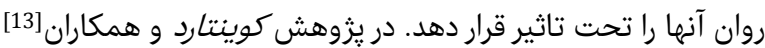

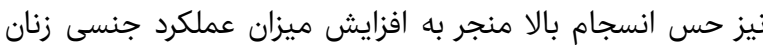
مبتلا به سرطان شد. ظرفيت هاى روانشناختى از جمله اميدوارى، تابآورىى، خوشبينى

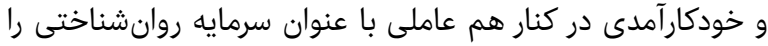

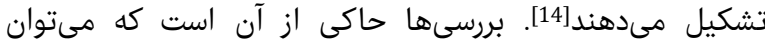

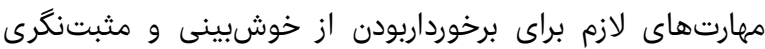

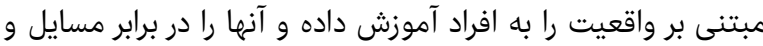

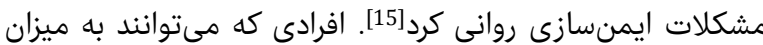

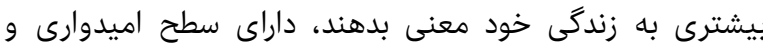

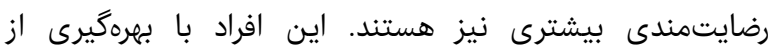

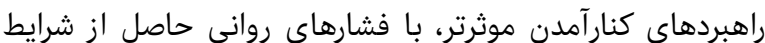

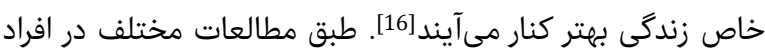

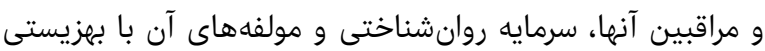

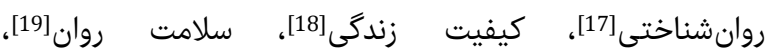
خودكارآمدى[20] و عملكرد شغلى[21] رابطه مثبت و معنى معندارى

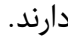

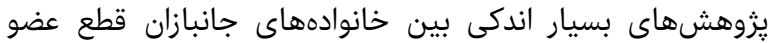

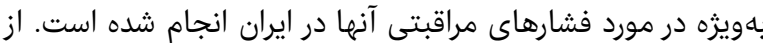

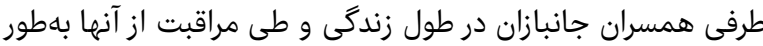

ييشبينى فشار مراقبت براساس حس انسار انسجام و

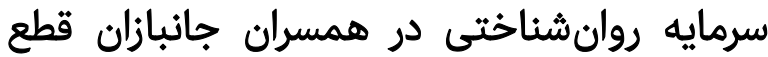

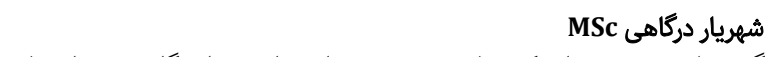
كروه علوم تربيتى، دانشكده علوم اهرم تربيتى و روانشناسى، دانشكاه محقق اردبيلى،

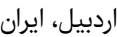

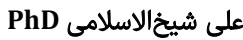

كروه علوم تربيتى، دانشكده علوم تربيتى و روانشئناسى، دانشكاه محقق اردبيلى،

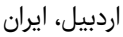

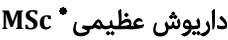

كروه علوم تربيتى، دانشكده علوم تربيتى و روان شناسى، دانشكاه محقق اردبيلى،

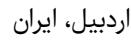

MSc اسماعيل شوانى إنشان

كروه مشاوره، دانشكده علوم تربيتى و روانشناسى، دانشكاه خوارزمى، تهران، ايران

جكيده

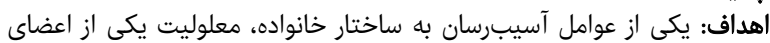

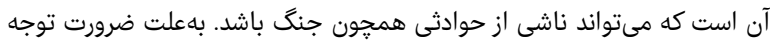

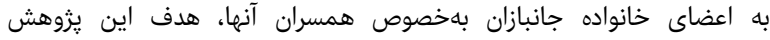

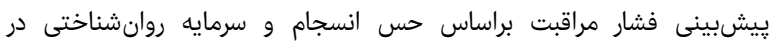
همسران جانبازان قطع عضو بود بردار

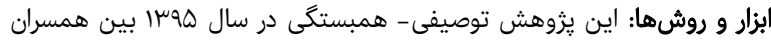

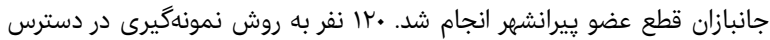

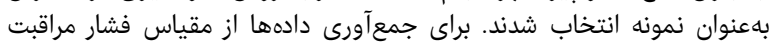

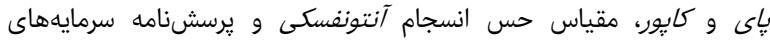

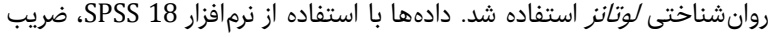

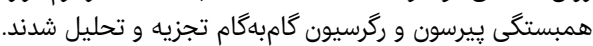

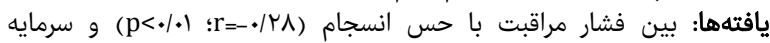

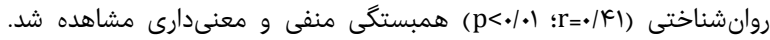

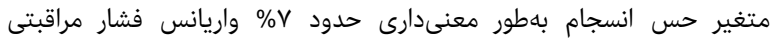

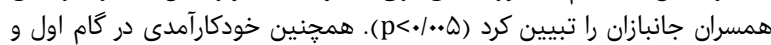

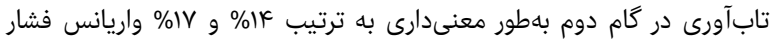

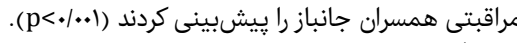

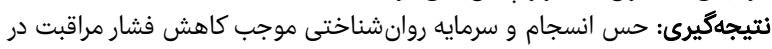

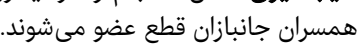
كليدوازهها: حس انسجام، همسران، قطع عضو، جاندون جانبازان

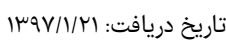

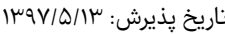

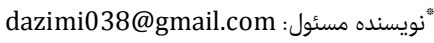

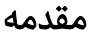

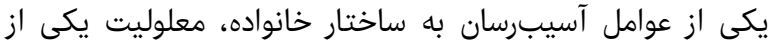

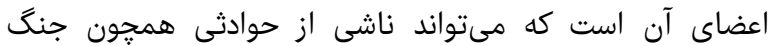

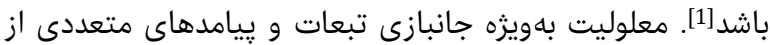

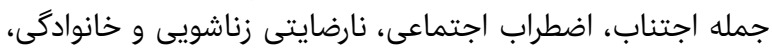

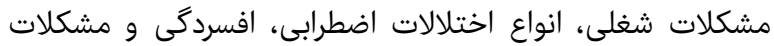

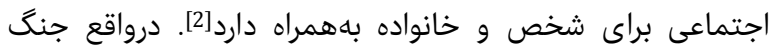

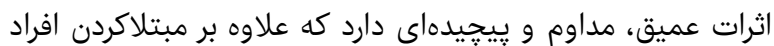

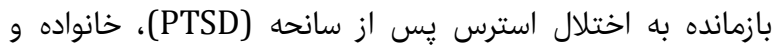

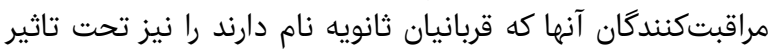

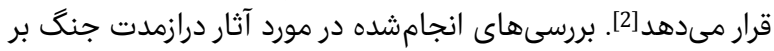

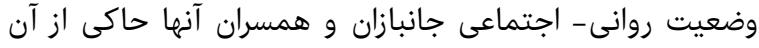

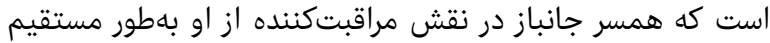

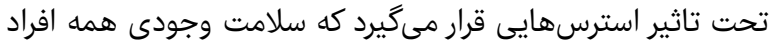

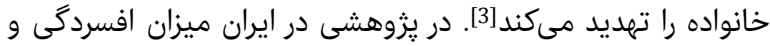




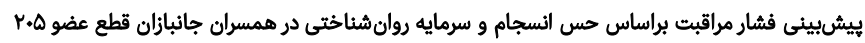

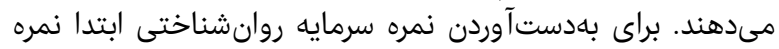

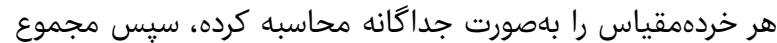

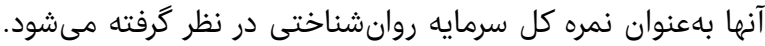

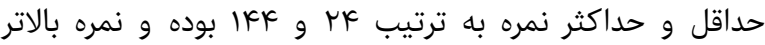

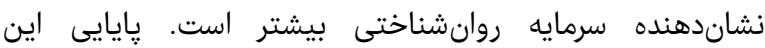

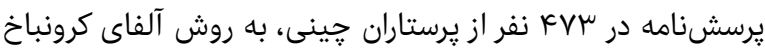
1/F

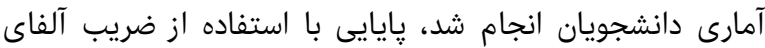

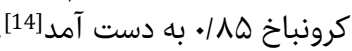

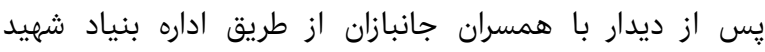

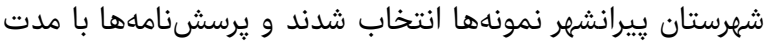

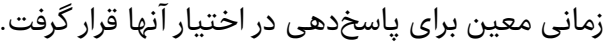

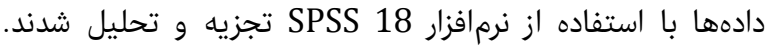

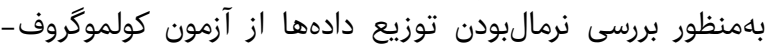

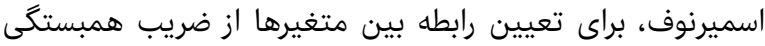

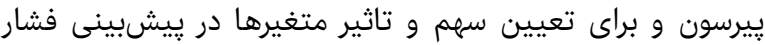

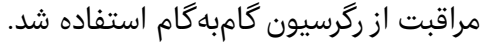

يافتهها

افراد شركتكننده داراى ميانكين سنى

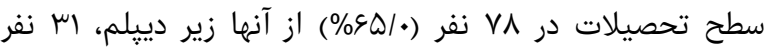

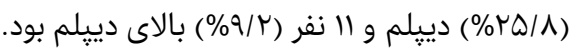

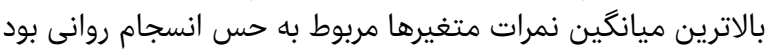

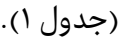

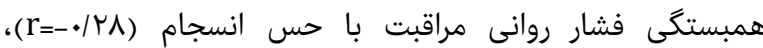

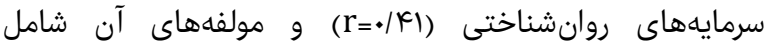

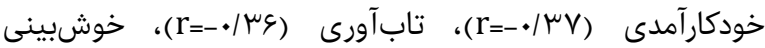

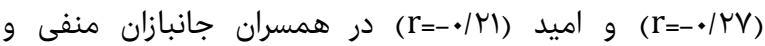

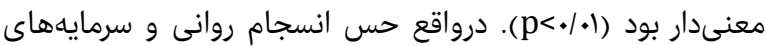

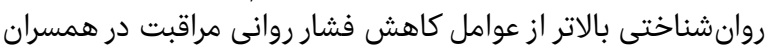

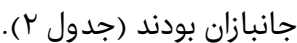

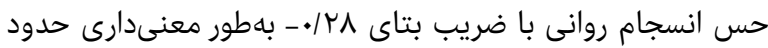

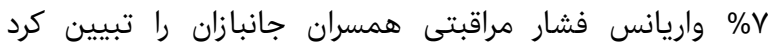

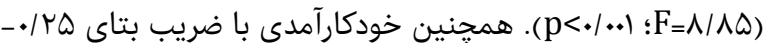

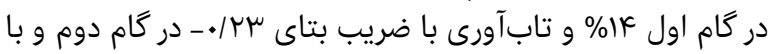

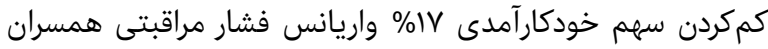

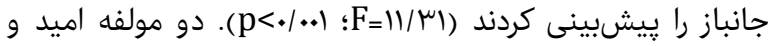

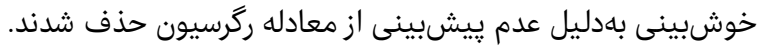

جدول () ميانكين آمارى نمرات متغيرهاى سرمايه روان شناختى و مولفههاى

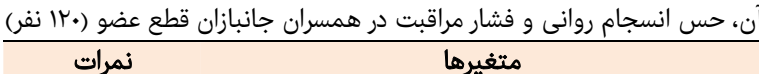

\begin{tabular}{|c|c|}
\hline & سرمايه روانشناختى \\
\hline$r F / F \mid \pm \mu / F \Delta$ & خودكارآمدى \\
\hline$r r / \Delta q \pm r / Q 1$ & تابآورى \\
\hline$r F / \Lambda q \pm \mu / r \mid$ & خوشبينى \\
\hline$r r / V q \pm \mu / K F$ & اميد \\
\hline$\Lambda \mu / \kappa \psi \pm q / \Lambda$. & كل \\
\hline $\mid \angle Q / Y I \pm N / \varphi q$ & كل حس انسجام روانى \\
\hline N/VQ $\pm r / K \varepsilon$ & كل فار مراقبت \\
\hline
\end{tabular}

مستقيم با فشارها و استرسهاى متعددى مواجه هستند. بنابراين

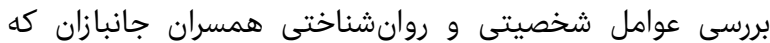

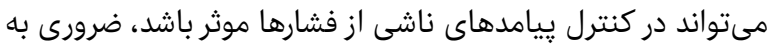
نظر مىرسد. يزوهش حاضر با هدف ييشبرسينى فشار مراقبت براساس حس

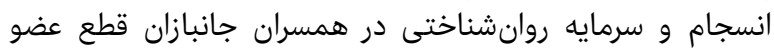

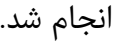

\section{ابزار و روشها} اين يزوهش توصيفى از نوع همبستخى است كه در سال هوسا بين

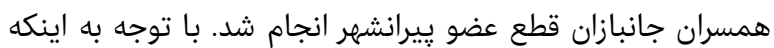

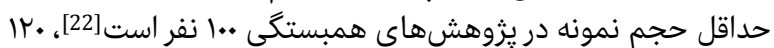

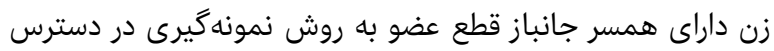

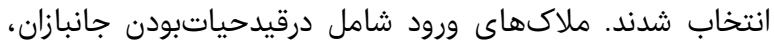

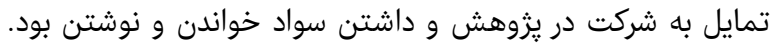

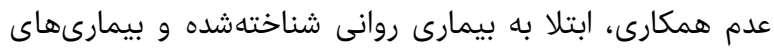

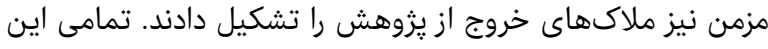

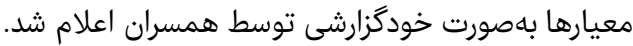

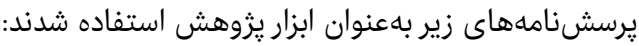

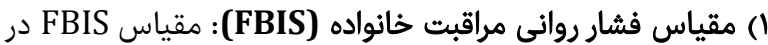

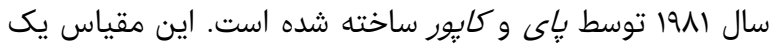

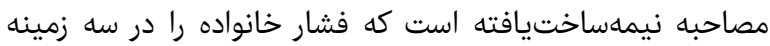

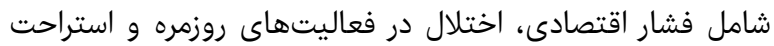

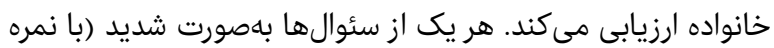

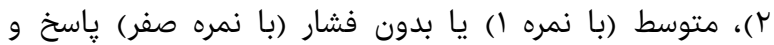

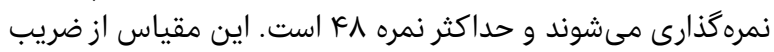

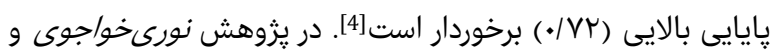

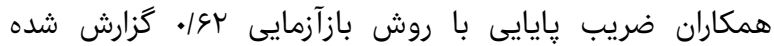
است]23].

r) مقياس حس انسجام روانى: فرم كوتاه مقياس حس انسجام

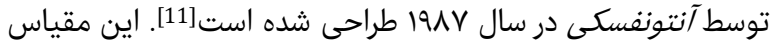

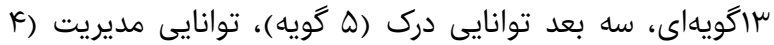

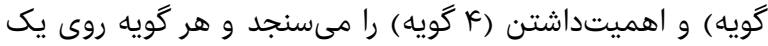

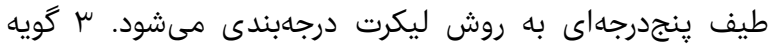

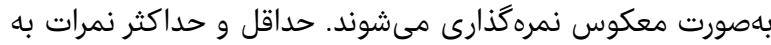

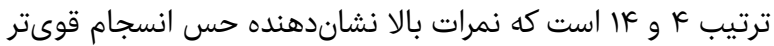

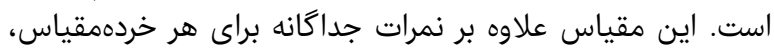

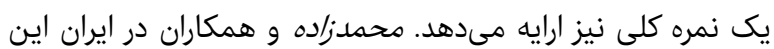

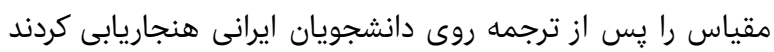

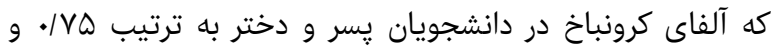

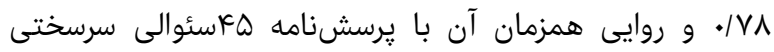

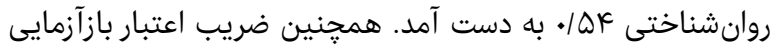

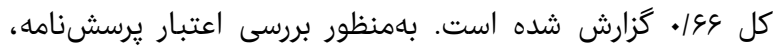

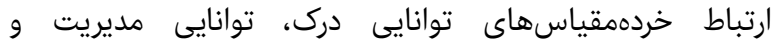

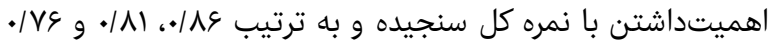

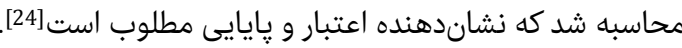

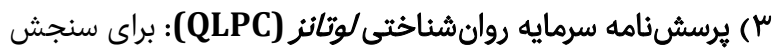

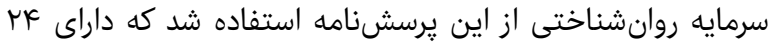

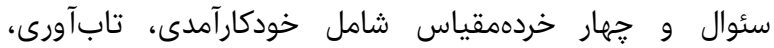

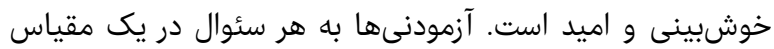

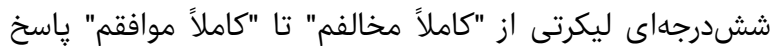


روانشناختى بالا در برابر آسيبها و عوامل استرسزاى غيرقابل

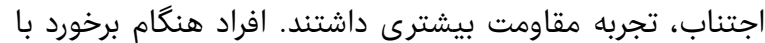

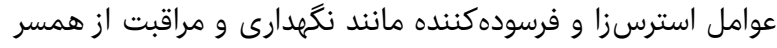

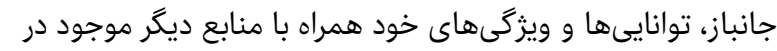

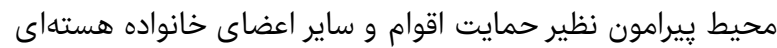

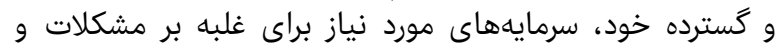

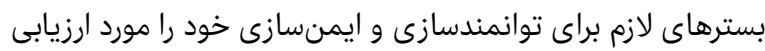

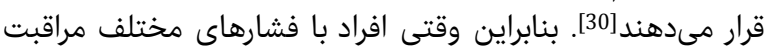

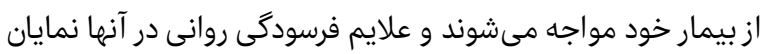

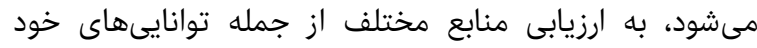

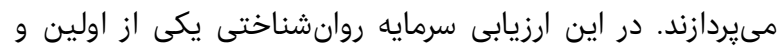

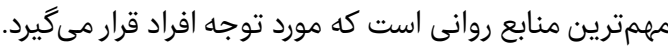

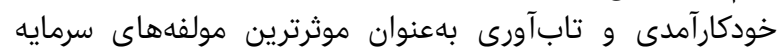

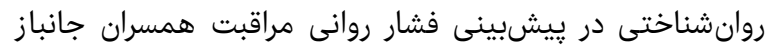

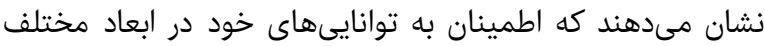

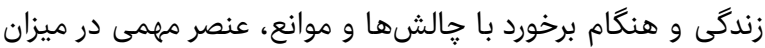

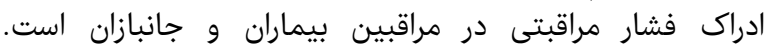

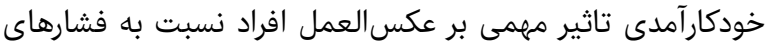

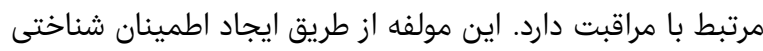

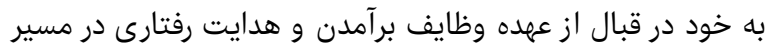

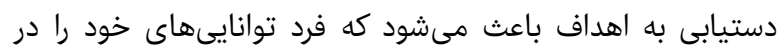

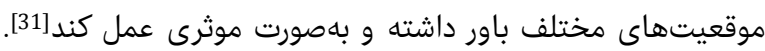

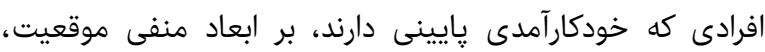

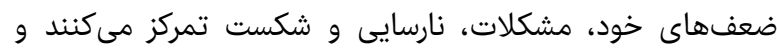

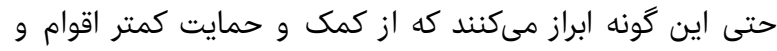

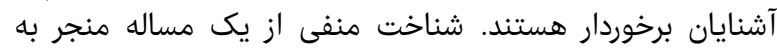

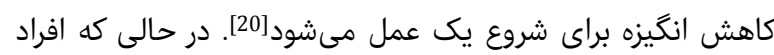

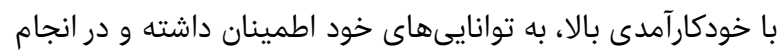

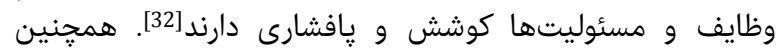

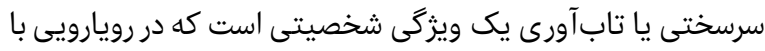

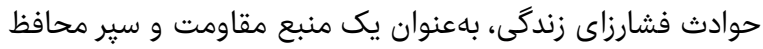

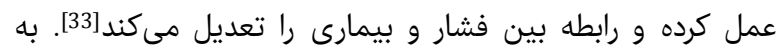

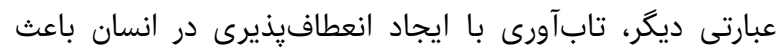

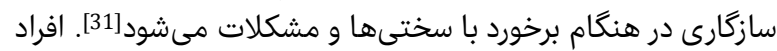

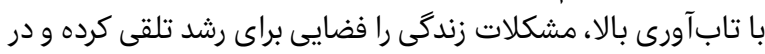

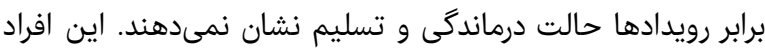

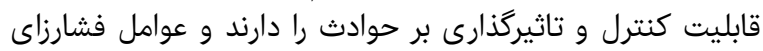

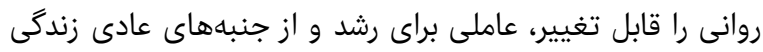

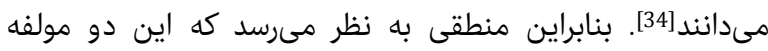

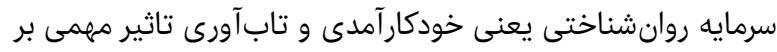

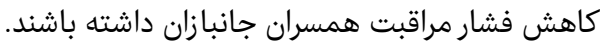

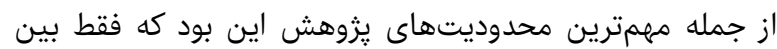

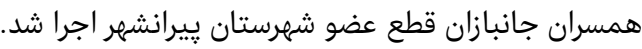

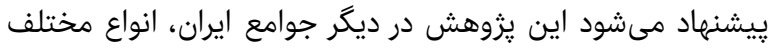

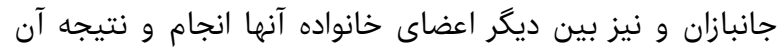

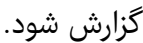

\section{نتيجه انيرى}

حس انسجام و سرمايه روانشناختى موجب كاهش فشارهاي روانى مراقبت در همسران جانبازان قطع عضو مى شوند.

تشكر و قدردانى: از همه افراد بهخصوص خانواده و همسران

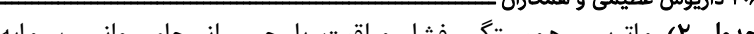

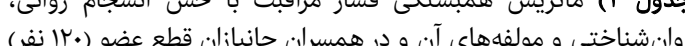

\begin{tabular}{|c|c|c|c|c|c|c|}
\hline 8 & $\Delta$ & $F$ & $\mu$ & $r$ & 1 & متغيرها \\
\hline & & & & & 1 & 1- فشار مراقبت كل \\
\hline & & & & & $\begin{array}{l}-. / \mu V^{\circ 0} \\
-. / \varphi^{\circ 0}\end{array}$ & ץ- - خابودكارآمدى \\
\hline & & 1 &.$/ \Delta \phi^{\circ \circ}$ &.$\Delta \Delta^{\circ}$ & $-0 / \pi Y^{\circ 0}$ & א - خوشبيني \\
\hline & & $\mid \Delta K^{\circ}$ & $.499^{\circ}$ & 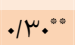 &.$- / \mu^{\circ}$ & هـ اميد \\
\hline &.$/ \mu k$ & $.4 . *$ & . $k \mu$ &. $\mid k *^{*}$ & $-0 / \pi \Lambda^{\circ}$ & 9-حس انسجام روانى كل \\
\hline
\end{tabular}

\section{بحث}

يزوهش حاضر با هدف يِيشبينى فشار مراقبت براساس حس حس

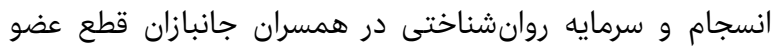

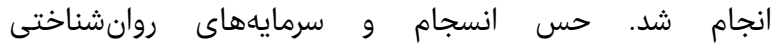
ييشبينى كنندهائ قوى و معنىدارى براى تبيين فشار مراقبت در

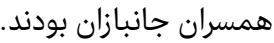

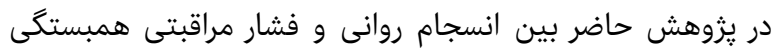

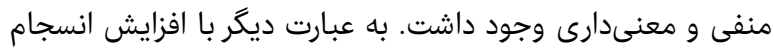

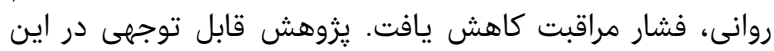

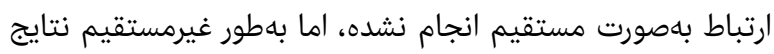

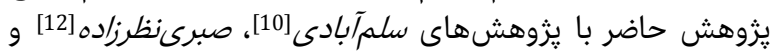

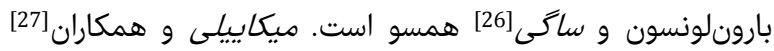

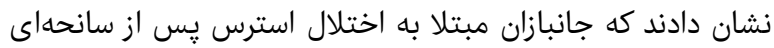

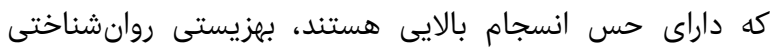

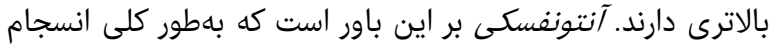

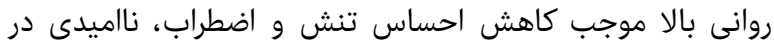

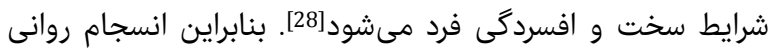

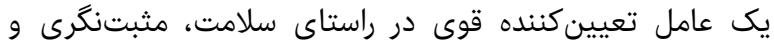

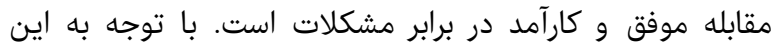

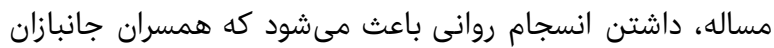

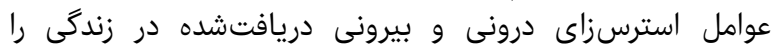

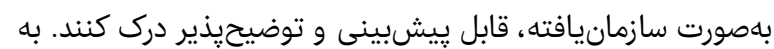

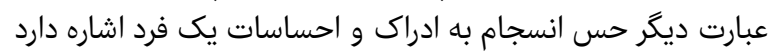

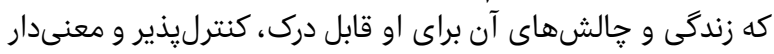

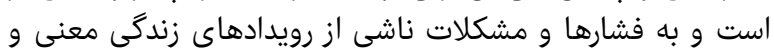

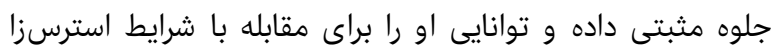

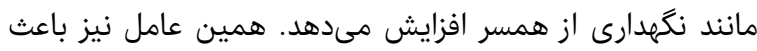

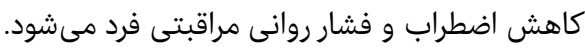

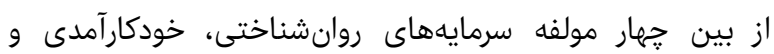

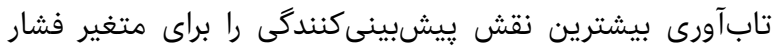

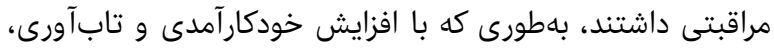

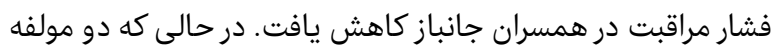

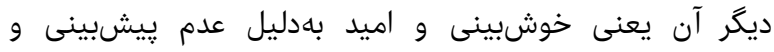

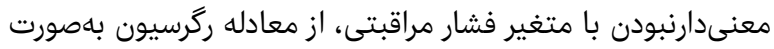

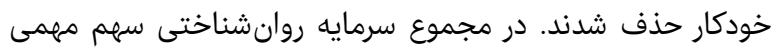

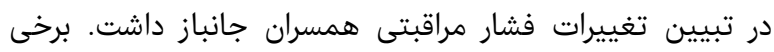

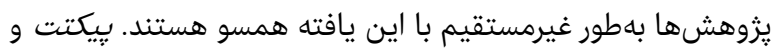

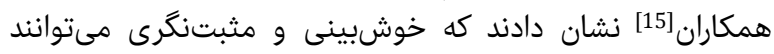

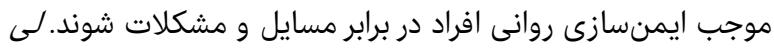

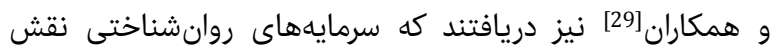

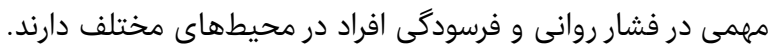

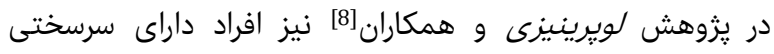




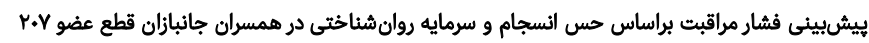

11- Antonovsky A. The structure and properties of the sense of coherence scale. Soc Sci Med. 1993;36(6):72533.

12- Sabri Nazarzadeh R, Abdkhodaei MS, Tabibi Z. Study of causal relationship between sense of coherence, psychological hardiness, coping strategies and mental health. Res Psychol Health. 2012;6(1):26-35. [Persian]

13- Quintard B, Constant A, Lakdja F, Labeyrie-Lagardère H. Factors predicting sexual functioning in patients 3 months after surgical procedures for breast cancer: The role of the sense of coherence. Eur J Oncol Nurs. 2014;18(1):41-5.

14- Soltani Z, Sadegh Mahboob S, Ghasemi Jobaneh R, Yoosefi N. Role of psychological capital in academic burnout of Students. Educ Strategy Med Sci. 2016;9(2):156-62. [Persian]

15- Pictet A, Coughtrey AE, Mathews A, Holmes EA. Fishing for happiness: The effects of generating positive imagery on mood and behaviour. Behav Res Ther. 2011;49(12):855-91.

16- Khodadadi Sangdeh J, Haghani M, Taheri M, Rezaiee Ahvanuee M, Ranjgar P. The relationship between the hopefulness, daily spiritual experiences and quality of life among the students. Community Health. 2014;9(2):38-48. [Persian]

17- Neyssi A, Arshadi N, Rahimi E. The causal relationship between psychological capital and positive emotions, psychological well-being, job performance and job engagement. J Psychol Achiev. 2011;4(1):19-46. [Persian]

18- Rahnema S, Baghersad Z, Shirazi M, Chitsaz A, Etemadifar M. Association between family caregivers' quality of life and psychological capital of the patients with multiple sclerosis. J Isfahan Med Sch. 2016;34(377):335-40. [Persian]

19- Ghaffari F, Fotokian Z, Rostami M, Meskini L. Analytical on effect of resilience interventions on mental health in family caregivers of elderlies with alzheimer's disease: A review literature. Clin Exc. 2017;7(1):13-21. [Persian]

20- Mohammadi Shahbalaghi F. Self- efficacy and caregiver strain in alzheimer's caregivers. Salmand Iran J Ageing. 2006;1(1):26-33. [Persian]

21- Khajepoor N, Beshlide K, Baharloo M. The relation between psychological capital and the exit of job, burnout, and job performance: The role of mediating job stress. J Ind Organ Psychol Stud. 2015;2(2):61-82. [Persian]

22- Soltani Z, Sadegh Mahboob S, Ghasemi Jobaneh R, Yoosefi N. Role of psychological capital in academic burnout of students. Educ Strategy Med Sci. 2016;9(2):156-62. [Persian]

23- Noori Khajavi M, Ardeshirzadeh M, Dolatshahi B, Afgheh S. Comparative evaluation of psychological burden in caregivers of patients with chronic schizophrenia and caregivers of patients with chronic bipolar disorder. Iran J Psychiatry Clin Psychol. 2008;14(1):80-4. [Persian]

24- Mahammadzadeh A, Poursharifi H, Alipour A. Validation of Sense of Coherence (SOC) 13-item scale in Iranian sample. Procedia Soc Behav Sci. 2010;5(WCPCG2010):1451-5.

25- Bayani AA, Bayani A, Rajabi A. The relationship between psychological capital, hope for employment and academic burnout with mental health among students of Islamic Azad University-Azadshahr Branch: A path
جانبازان گرامى كه ما را در اجراى اين يزوهش يارى كردند نهايت

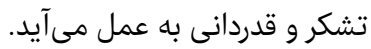

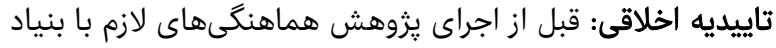

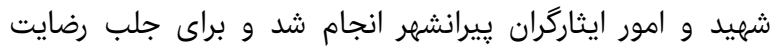

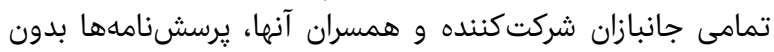

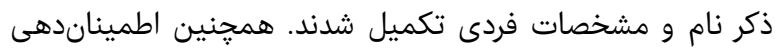

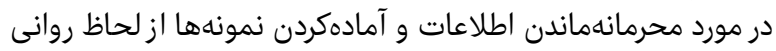

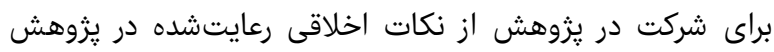
است. تعارض منافع: موردى از سوى نويسندكان بيان نشده است.

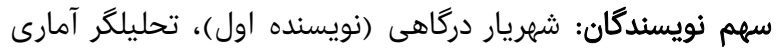

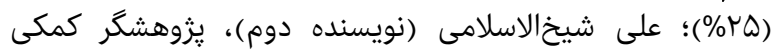

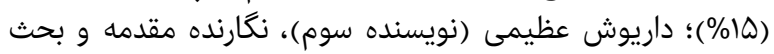

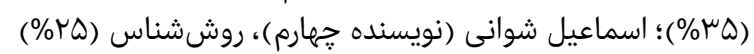
منابع مالى: كليه منابع مالى از طرف نويسندگًان تامين شده است.

منابع

1- Behdani F, Fayyazi Bordbar MR, Hebrani P, Talebi M, Kohestani L. Psychiatric symptoms in chemical and somatic combat veterans. J Mil Med. 2009;11(1):31-5. [Persian]

2- Hojjat SK, Talebi MR, Zamirinejad S, Shakeri M. The effectiveness of training stress coping strategies on marital conflict in wives of patients with post-traumatic stress disorder. J North Khorasan Univ Med Sci. 2012;4(2):185-90. [Persian]

3- Safavi M, Mahmoodi M, Akbarnatajbishe K. Assessment of relationship quality of life and coping skills in spouses of chemical devotees with pulmonary complications due to sulfur mustard in Tehran in 2006. Daneshvar Med. 2009;16(80):43-52. [Persian]

4- Manteghi A. Level of expressed emotion, depression and caregiver burden in wives of veterans admitted in psychiatric ward and their relationship with readmissions. J Fundam Ment Health. 2010;12(45):4109. [Persian]

5- Saban KL, Mathews HL, Janusek LW. The relationship among depression and caregiver burden with salivary cortisol awakening level in female caregivers of stroke survivors. Brain Behav Immun. 2010;24(Suppl1):S21.

6- Renshaw KD. Psychological and marital distress in spouses of Vietnam veterans: Importance of spouses' perceptions. J Anxiety Disord. 2010;24(7):743-50.

7- Dekel R, Monson CM. Military-related post-traumatic stress disorder and family relations: Current knowledge and future directions. Aggress Violent Behav. 2010;15(4):303-9.

8- Loprinzi CE, Prasad K, Schroeder DR, Sood A. Stress Management and Resilience Training (SMART) program to decrease stress and enhance resilience among breast cancer survivors: A pilot randomized clinical trial. J Clin Breast Cancer. 2011;11(6):364-8.

9- Liukkonen V, Virtanen P, Kivimäki M, Pentti J, Vahtera J. Sense of coherence and attrition during four-year follow-up in cohorts of permanent and non-permanent Finnish employees. BMC public health. 2008;8(1):1-7.

10- Salmabadi M, Rajabi MJ, Safara M. Effectiveness of training the review of life on life satisfaction and sense of coherence middle-aged women and elderly nursing home residents in Qazvin. Iran J Ageing Salmand. 2018;13(2):198-209. [Persian] 
Rostampour F, Asadi Z. Quality of life of the spouses of war related amputees of hamedan city. Iran J War Public Health. 2016;8(3):157-63. [Persian]

31- Luthans F, Avey JB, Avolio BJ, Peterson SJ. The development and resulting performance impact of positive psychological capital. Hum Resour Dev Q. 2010;21(1):41-67.

32- Azimi D, Ghadimi S, khazan K, Dargahi SH. The Role of Psychological Capitals and academic motivation in academic vitality and decisional procrastination in nursing students. J Med Edu Dev. 2017;12(3):147-57. [Persian]

33- Parsafar S, Yazdkhasti F. Investigating causal relationships between gender roles, resilience, hardiness, learned helplessness and co-dependency, in wives of addicted people. Women Stud. 2015;12(4):6992. [Persian]

34- Babaeiamiri N, Haghighat Dost S, Ashori J. The relationship of job burnout, perceived social support and psychological hardiness with mental health among nurses. Sci J Hamadan Nurs Midwifery Fac. 2016;24(2):120-8. [Persian]

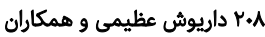
analysis. J Health Promot Manag. 2016;5(5):40-7. [Persian]

26- Braun-Lewensohn 0, Sagy S. Community resilience and sense of coherence as protective factors in explaining stress reactions: Comparing cities and rural communities during missiles attacks. Community Ment Health J. 2014;50(2):229-34.

27- Mikaeili N, Einy S, Tagavy R. Role of self-compassion and sense of coherence and thought action fusion in prediction of psychological well-being among posttraumatic stress disorder veterans. Iran J War Public Health. 2017;9(1):33-9. [Persian]

28- Valtonen M , Raiskila T, Veijola J, Läksy K, Kauhanen ML, Kiuttu J, et al. Enhancing sense of coherence via early intervention among depressed occupational health care clients. Nord J Psychiatry. 2015;69(7):515-22.

29- Li X, Kan D, Liu L, Shi M, Wang Y, Yang X, et al. The mediating role of psychological capital on the association between occupational stress and job burnout among bank employees in China. Int J Environ Res Public Health. 2015;12(3):2984-3001.

30- Naderi A, Sedighi S, Roshanaei G, Ahmadpanah M, 